\title{
La implicación de la familia en la comunidad escolar: gestación de una herramienta para la medición de percepciones cualitativas
}

\author{
Francesca Burriel \\ Universitat Autònoma de Barcelona. España. \\ francesca.burriel@uab.cat
}

Recibido: $13 / 2 / 2021$

Aceptado: $22 / 7 / 2021$

Publicado: 31/1/2022

\section{Resumen}

La comunidad escolar se ha definido tradicionalmente como el conjunto de personas que interaccionan en un mismo ecosistema educativo, usualmente una escuela, en el que se desarrolla una acción intencionada, global y contextualizada hacia la enseñanza y el aprendizaje de los significados, los procesos y los valores de una sociedad (Mateos y Peñalba, 2003). Desde una perspectiva jurídica, la participación de todos los implicados constituye un derecho y una responsabilidad ciudadana irrenunciable, vinculada a derechos fundamentales como la educación, la dignidad humana, la seguridad jurídica o la protección a la infancia (UNESCO, 2014). En este artículo se presenta una herramienta conceptual para la triangulación de opiniones que permite utilizar la suma de visiones muy diversas y cuantificar elementos mínimos de información, transformando las opiniones cualitativas (qualia) en unidades binarias (quantum) que se pueden cuantificar, comparar y gestionar informáticamente. A partir del método de investigación-acción los resultados de las investigaciones realizadas han permitido dar voz al alumnado, al profesorado, a las familias, a los directivos y al personal de la administración del centro, en un ejercicio de autoevaluación y transparencia en la rendición de cuentas. En un ecosistema territorial más amplio también se podría incorporar en el análisis las voces de otros agentes educativos del territorio.

Palabras clave: comunidad escolar; herramienta de análisis; alumnado; familias; participación

Resum. La implicació de les famílies en la comunitat escolar: gestació d'una eina per mesurar percepcions qualitatives

La comunitat escolar ha estat definida tradicionalment com el conjunt de persones que interaccionen en un mateix ecosistema educatiu, usualment una escola, on es desenvolupa una acció intencionada, global i contextualitzada cap a l'ensenyament i l'aprenentatge dels significats, els processos i els valors d'una societat (Mateos y Peñalba, 2003). Des d'una perspectiva jurídica, la participació de tots els implicats constitueix un dret i una responsabilitat ciutadana irrenunciable, vinculada a drets fonamentals com ara l'educació, la dignitat humana, la seguretat jurídica o la protecció de la infancia (UNESCO, 2014). En aquest article es presenta una eina conceptual per a la triangulació d'opinions que permet utilitzar la suma de visions molt diverses i quantificar elements mínims d'infor- 
mació, transformant la coincidència de les opinions qualitatives (qualia) en unitats binàries (quantum) per poder-les quantificar, comparar i gestionar informàticament. A partir del mètode d'investigació-acció els resultats de l'anàlisi realitzada han permès donar veu a l'alumnat, al professorat, a les famílies, als directius i al personal de l'administració del centre, en un exercici d'autoavaluació i transparència en la rendició de comptes. En un ecosistema territorial més ampli, també es poden incorporar a l'anàlisi les veus d'altres agents educatius del territori.

Paraules clau: comunitat escolar; eina d'anàlisi; alumnat; famílies; participació

\section{Abstract. Family engagement in the educational community: Development of a tool for measuring qualitative perspectives}

The educational community has traditionally been defined as the set of people who interact within an educational ecosystem, usually a school or structured educational environment, in which an intentional, global and contextualized action occurs towards the teaching and learning of meanings, processes and values of a society (Mateos y Peñalba, 2003). From a legal perspective, the participation of all those involved constitutes an inalienable civic right and responsibility, linked to fundamental rights such as education, human dignity, legal security and protection of children (UNESCO, 2014). This article presents a conceptual tool for triangulating opinions. It allows the range of very different perspectives to be included, and the slightest elements of information to be quantified, transforming qualitative opinions (qualia) into binary units (quantum) that can be quantified, compared and managed. By using the research-action method, the research results have given students, teaching staff, families, school principals and administrative staff a voice, in an exercise of self-evaluation and transparency for accountability. In a wider ecosystem, voices from other agents linked to the educational community can be also incorporated into the analysis.

Keywords: school community; analysis tool; students; families; participation
Sumario
1. Introducción
4. Resultados
2. Los objetivos: la gestación de una
5. Conclusión y reflexiones
herramienta de análisis
Referencias bibliográficas

3. El método: estudio realizado

\section{Introducción}

En un mundo en continua transformación, los sistemas educativos se enfrentan a retos prácticos y conceptuales difíciles de gestionar si no se les incluyen las voces de todos los implicados (Fernández-Rouco, Fallas-Vargas y GarcíaMartínez, 2020). En las últimas décadas hemos visto cómo se incorporaban a la escuela los procesos de evaluación interna, la opinión de las familias sobre el cambio educativo o incluso la participación del alumnado en la definición de sus propias tareas escolares. Los diferentes agentes de la comunidad educa- 
tiva muestran con frecuencia una enorme diversidad de objetivos, prioridades y elementos cualitativos no siempre fáciles de coordinar y ajustar.

Los individuos y los colectivos se comportan de forma distinta, gozan de percepciones diferentes y realizan valoraciones muy diversas acerca de las cosas que perciben (Fernández Batanero, 2012; Dietze, 2021). Los miembros de la comunidad escolar pueden tener distintos roles y grados de influencia en el proceso colectivo (Peñaloza Páez, 2012), de manera que la interacción entre ellos, las mecánicas de organización académica y las esperanzas de futuro determinan también diferentes entornos educativos y climas de aprendizaje. El espacio escolar (Zardoya, 2015), las vías de participación (Martín y Muñoz, 2006), los agentes implicados (Astudillo y Chévez, 2014), las directrices educativas (Niño y Gama, 2014) y las interacciones de los agentes de la comunidad escolar (Somprach, Tang y Popoonsak, 2017) configuran un escenario dialéctico necesario e incluso indispensable para identificar la realidad compleja de la comunidad educativa. Para abordar esta diversidad multimodal es necesario disponer de estrategias de análisis basadas en la reducción de información y la aplicación de constructos metacognitivos que permitan transferir las opiniones cualitativas de los protagonistas a datos operables para facilitar la gestión matemática de la información. En este artículo se presenta un modelo utilizado por la autora en estudios de pedagogía aplicada, en la Universidad Autónoma de Barcelona (UAB), que permite abordar diversos campos de estudio y alinearlos con los marcos analíticos de la OCDE en educación, la Agenda 2030 de las Naciones Unidas (Naciones Unidas, 2015) y la declaración de Incheon para la transformación de la educación (UNESCO, 2015a). Entender la educación como un bien común (UNESCO, 2015b) no puede ser meramente una intención o un principio teórico, sino que se debe entender como un proceso participativo de responsabilidad colectiva, donde cada uno ha de tener su papel y su responsabilidad.

La educación cambia vidas, y participar en los procesos de mejora educativa puede llegar a transformar comunidades y sociedades enteras (UNESCO, 2017). Por otro lado, la investigación actual, tal vez por influencia de los procesos participativos en las redes sociales, de likeldislike, se limita a veces a pedir la opinión favorable o desfavorable de la audiencia, confundiendo esta respuesta espontánea con un auténtico proceso de participación, que debe por su parte entenderse como un derecho a decidir, proponer tendencias de futuro y compartir horizontes negociados y acordados colectivamente. Pasar de un «me gusta / no me gusta» o un «estoy de acuerdo / no estoy de acuerdo» a una cifra cuantificable (1-0) suele ser relativamente fácil, pero determinar distintos rangos cuantitativos de complejidad sobre conceptos difusos (por ejemplo: la calidad del centro, los procesos comunicativos, la participación de las familias o el bienestar del alumnado) a menudo cae en la simple escala de opinión o en el grado de aceptación difícilmente objetivable. Por el contrario, una herramienta validada que sea capaz de distinguir niveles de complejidad cognitiva y de abstracción conceptual para identificar diferentes aspectos a duras penas mesurables de las comunidades educativas nos da una granularidad mucho más fina 
en lo que se refiere a la comprensión de realidades de percepción múltiple. Si además los niveles resultantes presentan una alineación con índices internacionales de medición de resultados, la herramienta puede facilitar estrategias de evaluación interna en centros educativos, o de valoración personal del liderazgo directivo, a partir de la gestión del conocimiento colectivo de componentes, mecánicas y dinámicas concretas.

En este estudio se facilita una descripción del proceso de elaboración de una evaluación de centro educativo desde la construcción colectiva del concepto participación de las familias en la comunidad educativa a partir de un método mixto (cualitativo y cuantitativo). Asimismo, se presenta una breve aplicación práctica llevada a cabo durante dos cursos escolares (2019-2020 y 2020-2021) en tiempos de pandemia, donde el rol parental ha variado sustancialmente en periodos distintos del curso, en función de la situación sanitaria del territorio y de las directrices legales dictadas en cada momento.

\section{Los objetivos: la gestación de una herramienta de análisis}

El presente estudio ofrece una herramienta de análisis multimodal que permita analizar realidades complejas a partir de la valoración de los distintos protagonistas, construyendo valores mesurables desde el constructo cognitivo y los niveles cualitativos de abstracción utilizados por cada informante. En el ámbito educativo se centra en la elaboración de un marco conceptual que define la participación de las familias como el objeto de estudio a identificar y mesurar, más allá del «sí/no» o del habitual «defina usted del 0 al 5».

Para ello, se recoge la propuesta ternaria de la OCDE sobre la construcción de los marcos de evaluación de las pruebas PISA que actualmente determinan buena parte de las políticas educativas del mundo global (Grek e Ydesen, 2021). Los tres ámbitos conceptuales que utiliza habitualmente la OCDE en sus marcos analíticos (niveles explícito, implícito y metarreferencial) permiten ajustar las preguntas de complejidad variable en las pruebas PISA de cualquier disciplina o ámbito evaluado (OECD, 2018). Para expresar de forma sencilla y unificada los tres niveles definidos en distintos marcos internacionales utilizados en la actualidad, podemos hacer referencia a:

Nivel 1: conocimiento explícito o demostrativo. Conocer los elementos, los conceptos o los contenidos fenomenológicos identificables, inventariables o susceptibles de operar e interactuar para definir una realidad.

Nivel 2: conocimiento implícito o procedimental. Organizar, explicar, analizar y comparar los procesos y las relaciones que se dan entre elementos o realidades concretas y los cambios y las tendencias de la realidad a partir de la intervención humana.

Nivel 3: conocimiento metarreferencial o integrado. Valorar justificadamente, proponer hipótesis y aportar interpretaciones creativas e innovadoras para favorecer modelos de transformación que tengan en consideración el bien común desde una perspectiva colectiva ética y sostenible. 
Estos tres niveles cognoscitivos se basan a su vez en los seis estadios de la conocida pirámide de Benjamin Bloom (1956), que permiten al docente y al investigador aproximarse a la realidad con criterios cualitativos, además de clasificar las distintas opiniones según la profundidad del conocimiento que se tenga de un hecho determinado. Sobre este mismo esquema, las nuevas teorías del juego han elaborado también un modelo en tres ámbitos análogos a los anteriores (Werbach y Hunter, 2012), representado en una pirámide de ludificación que mantiene una gran alineación con las categorías de abstracción operativas vistas anteriormente (figura 1 ).

Figura 1. Pirámide que unifica la propuesta de Werbach y Hunter (2012) y la jerarquía cognoscitiva de la OCDE en la competencia global (2018)

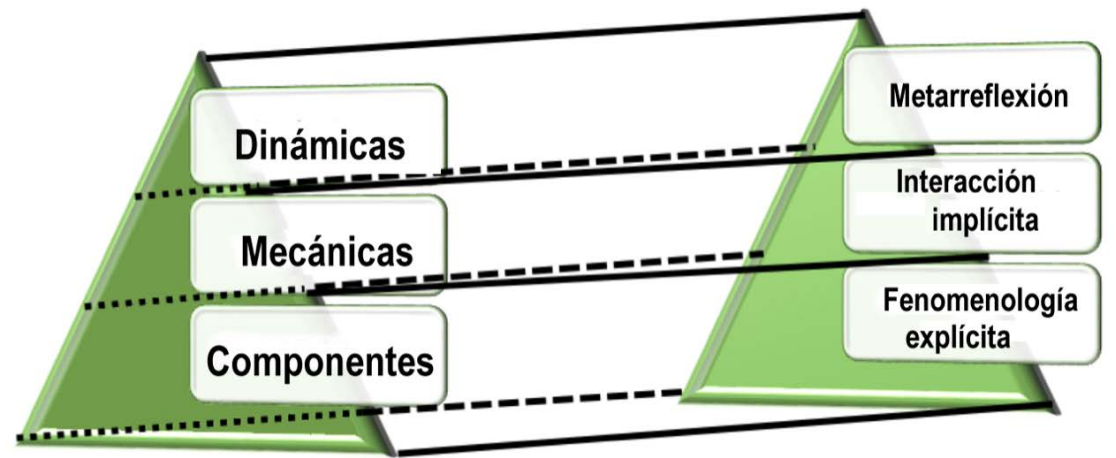

Fuente: adaptación de la autora, 2021.

Así, la investigación sobre el estado de la participación de las familias en un centro educativo puede realizarse a partir del análisis cualitativo de la opinión de padres, madres, alumnos o familiares, y también del registro de metadatos que identifique si en su discurso informativo se detecta información de nivel 1 (componentes), nivel 2 (mecánicas) y nivel 3 (dinámicas). Cuantificando la frecuencia en que aparecen las unidades de información de cada nivel obtenemos índices cuantificables (quantum) de variables cualitativas (qualia). Si se asigna un valor ponderado a cada ámbito cognoscitivo, se puede incluso definir el valor acumulado para cada intervención o discurso en su conjunto y evaluar la riqueza ontológica de temas y variables educativas muy diversas (tabla 1).

La información así analizada se puede recoger en fuentes muy diversas, tanto en situaciones estructuradas como no estructuradas (en entrevistas, cuestionarios abiertos, comunicación libre de informantes individuales o situaciones de interacción en grupo), y se puede identificar la calidad de la información, la participación o la toma de decisiones que llegan a tener las familias en el ámbito escolar a escala internacional (Verger, Novelli y Altinyelken, 2018). En 
Tabla 1. Matriz de construcción conceptual en tres ámbitos o niveles de abstracción para cuantificar el objeto de estudio, en correlación con otras clasificaciones internacionales del ecosistema educativo

\begin{tabular}{llll}
\hline $\begin{array}{l}\text { Propuesta de la autora: } \\
\text { matrices conceptuales de metadatos } \\
\text { en tres ámbitos y niveles de } \\
\text { abstracción }\end{array}$ & $\begin{array}{l}\text { Cognición de } \\
\text { Bloom revisada } \\
\mathbf{( 2 0 0 1 )}\end{array}$ & $\begin{array}{l}\text { Ludificación } \\
\text { de Werbach y } \\
\text { Hunter (2012) }\end{array}$ & $\begin{array}{l}\text { Marco } \\
\text { competencial de } \\
\text { la OECD (2018) }\end{array}$ \\
\hline $\begin{array}{l}\text { Ámbito de nivel 1: elementos del siste- } \\
\text { ma (recursos, normas, protagonistas...). }\end{array}$ & $\begin{array}{l}\text { Conocer } \\
\text { Ámprender }\end{array}$ & Componentes & $\begin{array}{l}\text { Conocimiento } \\
\text { explícito o demos- } \\
\text { trativo. }\end{array}$ \\
$\begin{array}{l}\text { Ámbito de nivel 2: procesos de interac- Utilizar } \\
\text { ción (procedimientos de gestión, desa- } \\
\text { rrollo, participación). }\end{array}$ & Analizar & Mecánicas & $\begin{array}{l}\text { Conocimiento } \\
\text { implícito o proce- } \\
\text { dimental. }\end{array}$ \\
\hline $\begin{array}{l}\text { Ámbito de nivel 3: actitudes transfor- Evaluar } \\
\text { madoras (tendencias de cambio, prin- } \\
\text { cipios de valor, narrativas y tendencias } \\
\text { de cambio, emociones prioritarias en la }\end{array}$ & Dinámicas & \\
toma de decisiones. & & $\begin{array}{l}\text { Conocimiento } \\
\text { metarreferencial o } \\
\text { integrado. }\end{array}$ \\
\hline
\end{tabular}

Fuente: comparación de la autora, 2021.

un proceso similar al que se genera con la aplicación de etiquetas o metadatos, la recogida de información de los distintos informantes, jerarquizada por la matriz generada en este proceso, ofrece una mayor precisión sobre la calidad del contenido en el análisis final. Este mismo análisis se ha aplicado a distintas esferas de interacción del ámbito escolar (comunicación entre la familia y la escuela, participación del alumnado) y se está llevando a cabo actualmente en estudios complementarios relacionados con «el uso de las evidencias de inclusión en el centro educativo", en el marco de un proyecto internacional Erasmus+ (EIPSI, 2021).

La posibilidad de cuantificar las frecuencias de «uso explícito de informaciones identificadas en una conversación determinada» sobre el tema de estudio (componentes identificables, mecánicas procedimentales y dinámicas estratégicas) abre un panorama extraordinario para la automatización del cálculo a partir de componentes básicos de información, fundamentado en el análisis de las opiniones de toda la comunidad educativa. Los cuestionarios de opinión censal adquieren en este caso un enorme potencial para la gestión de macrodatos (bigdata) y el análisis de tendencias a gran escala. La clasificación sectorial de esta información o la comparación y la triangulación entre centros, colectivos o territorios puede aportar un conocimiento mucho más detallado de la realidad que un simple cuestionario de opinión o una cuantificación acumulativa sin granularidad conceptual.

\section{El método: estudio realizado}

La participación de las familias en la comunidad educativa ha sido objeto de diversas investigaciones en las últimas décadas (Garreta, 2012; Jeynes, 2011; Kernan, 2012; Razeto 2016; Rivas y Ugarte, 2014; Aguilar, Demosthenes y 
Campos, 2020). Un estudio preliminar del estado del arte permite apreciar las tendencias que relacionan esta participación con la transparencia educativa, la democratización de la toma de decisiones y el reconocimiento de derechos fundamentales en la educación. En este contexto, el presente estudio se ha llevado a cabo virtualmente, en plena pandemia de COVID-19, y ha incorporado también el reconocimiento del papel de las familias en la educación escolar durante los periodos de confinamiento, así como reflexiones sobre la injusticia que representa la desigualdad de recursos en situaciones extremas como la vivida en los dos últimos cursos.

En el estudio bibliométrico y la investigación inicial y en el estado del arte se establece un aumento considerable de «la participación de las familias en la comunidad educativa», con reformulación de roles, diversidad de fuentes de información y flexibilización académica en ecosistemas híbridos (presenciales y digitales).

La ficha técnica del estudio puede resumirse en los siguientes parámetros:

a) Contexto académico: Departamento de Pedagogía Aplicada de la Facultad de Ciencias de la Educación, Universidad Autónoma de Barcelona.

b) Localización: Barcelona, Cataluña (España).

c) Objetivo 1: elaboración de una herramienta de análisis para conocer el grado de participación de las familias en el entorno escolar.

Para crear una matriz conceptual de análisis se ha generado un modelo de elaboración propia a partir de la pirámide de Bloom (1956), la pirámide de ludificación (Werbach y Hunter, 2012) y la jerarquía de los marcos analíticos educativos de la OECD (2018). Esta herramienta se ha aplicado en la elaboración de cuestionarios de opinión (a diversos sectores educativos), el análisis documental y los grupos de debate conjunto que se han utilizado como fuentes de información. Para que dichos cuestionarios tuviesen certidumbre y robustez se dispuso la aplicación del análisis de validez de contenido del modelo Lawshe (1975). El índice obtenido de acuerdo con la validación de los expertos fue ampliamente superior al 0,58 en todas las preguntas aquí referenciadas, por lo que se considera validado por la de la supervisión de los expertos competentes en la materia, que eran personas de reconocido prestigio y formación en el campo de la educación e ilustres por su reputación y consideración por la comunidad educativa (Jiménez, 2020).

Se realizan 20 preguntas a 64 representantes de cada uno de los sectores educativos seleccionados (profesores, alumnos, familias y equipo directivo), en un total de 8 escuelas de primaria de Barcelona (6 públicas y 2 concertadas, de titularidad privada sostenidas con fondos públicos). Las 1.280 respuestas se analizan, se comparan y se triangulan por sectores y escuelas, y se contrastan con los documentos oficiales de cada centro. Finalmente, los resultados se contrastan en grupos de debate con representantes de familias y docentes en cada escuela.

En los cuestionarios utilizados hay tres preguntas clave para identificar el estado de la relación comunicativa entre la familia y la escuela: «¿Cuál es el tema 
estrella que se trata en la primera reunión de la escuela con las familias?», «Cómo se escoge el delegado de las familias en el centro escolar?» y «Cuando existe un problema con un grupo de familias, ¿cuál es, según tu criterio, la mejor manera de abordarlo?».

Las respuestas muestran diferentes grados de madurez participativa entre las familias y la escuela (según su interés en información básica, en los procesos relacionales implícitos o en las dinámicas de transformación), que correlacionan considerablemente con los resultados educativos del centro en términos de competencias básicas. Esta correlación puede entenderse porque una mayor participación en la toma de decisiones por parte de las familias puede significar mayor implicación en el proceso educativo de sus hijos y por tanto ser relevante en los resultados académicos del alumnado. El desarrollo de la investigación se acompaña de una reflexión específica sobre el valor de las herramientas generadas y su relevancia para superar la subjetividad en el análisis de una realidad que surge de las opiniones agregadas de todos los participantes.

\section{Resultados}

El análisis de resultados del presente estudio permite identificar factores concretos que difieren o se repiten en diversas escuelas. La triangulación entre distintos sectores educativos aporta también una mayor riqueza informativa sobre la realidad de cada centro, y la comparación de los documentos oficiales del centro ajusta aún más la mirada multimodal en cada realidad. Las tres preguntas aquí seleccionadas permiten ilustrar los distintos ámbitos conceptuales de los cuestionarios, con aspectos que recogen información explicita sobre la implicación de las familias en el centro (como los temas de discusión en la reunión de inicio de curso), información procedimental (como la gestión de los representantes de padres y madres) e información relativa a la toma de decisiones en momentos de crisis o en situaciones que requieren cambios en la toma de decisiones.

Para la pregunta "¿Cuál es el tema "estrella" que se trata en la primera reunión con las familias?», el índice global mayoritario de respuestas apunta a temas concretos de tipo organizativo, de gestión de grupos y de ordenación escolar, donde coinciden todos los sectores. La segunda opción apunta hacia temas educativos en los que se incluyen aspectos personales y emocionales del alumno, como su bienestar personal, las conductas sociales y los soportes socializadores. Esta pregunta identifica temas concretos, usualmente definidos y de clasificación sencilla. Llama la atención la dispersión en la respuesta de los docentes y la dirección del centro, así como la mayor concentración entre las familias. Se trata, pues, de un ámbito en el que las madres y los padres enfocan sus intereses en el contexto escolar de forma ajustada y pertinente, aunque no necesariamente diversificada o imaginativa (figura 2). 
Figura 2. Respuestas de la muestra a la pregunta «¿Cuál es el tema "estrella" que se trata en la primera reunión con las familias?»

15

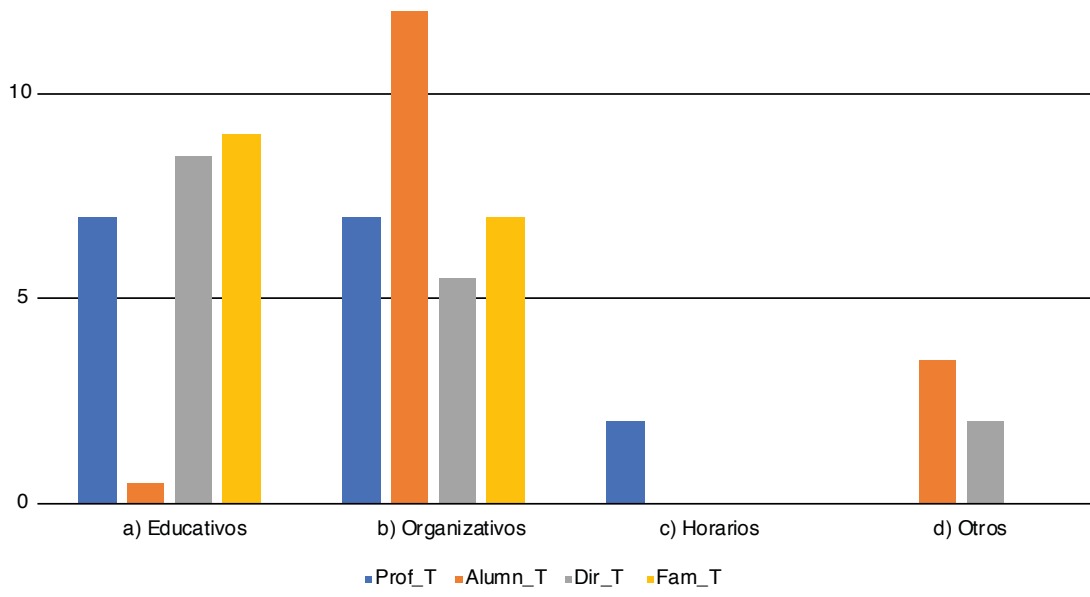

Fuente: elaboración propia.

La pregunta "¿Cómo se escoge el delegado de las familias en el centro escolar?» representa la expresión práctica de la implicación parental en el ámbito académico, con el ejercicio de sus derechos de representación y participación en la organización educativa. Con todo, los resultados son notoriamente insuficientes. En el análisis de las respuestas destaca la escasa votación o la selección por proceso delegatorio, mientras que la opinión mayoritaria apunta, en el apartado "otros», a todo tipo de nombramiento dirigido o determinado por el centro, también en los centros públicos (incluso «no hay representantes»), a pesar de que existe en el territorio una normativa explícita para seleccionar referentes y representantes en las escuelas públicas. La práctica democrática que ha de garantizar la implicación de las familias en el centro parece claramente infrautilizada, según las manifestaciones de todos los sectores educativos consultados (figura 3).

La tercera pregunta mostrada en este artículo representa un espacio de metarreflexión sobre la implicación de las familias en el centro, dado que hace referencia al tercer nivel de la matriz, esta vez generando criterios de valor y propuestas proyectivas: "Cuando existe un problema con un grupo de familias, ¿cuál es la mejor manera de abordarlo?». Esta cuestión permite descubrir opiniones referidas a momentos de crisis, cuando la toma de decisiones es esencial y se pone de manifiesto el protagonismo parental y sus expectativas, en situaciones en las que hay que abordar cambios en los usos participativos habituales. En esta extracción concreta se ha eliminado el alumnado, pero eso no evita 
Figura 3. Respuestas de la muestra a la pregunta «¿Cómo escoge el delegado de las familias en el centro escolar?»

20

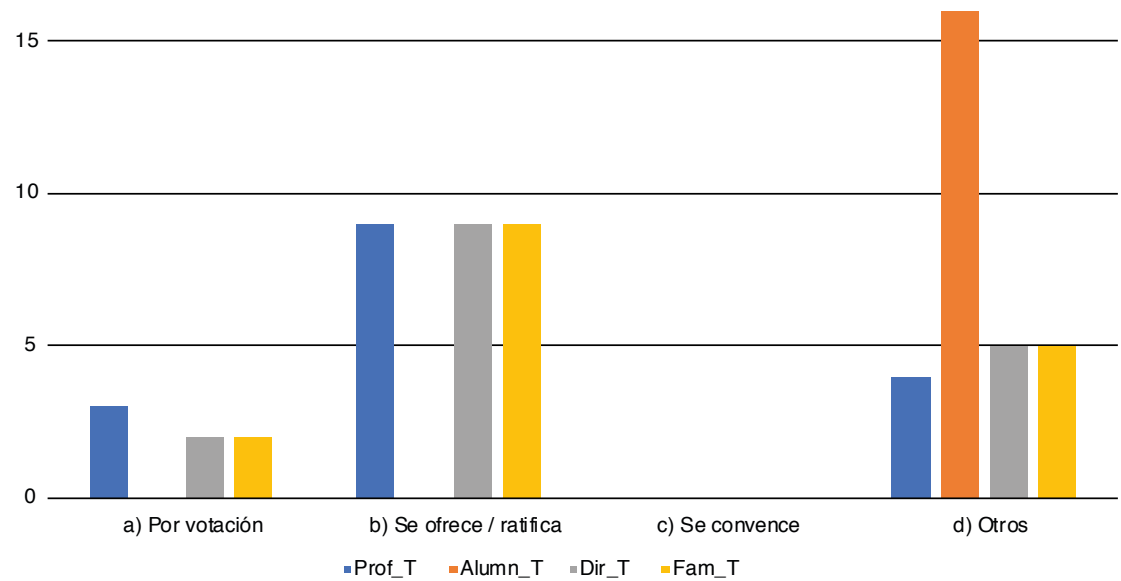

Fuente: elaboración propia.

una dispersión de opiniones visible entre las familias, mientras que el profesorado prefiere mayoritariamente abordar cada familia por separado en caso de conflicto (figura 4).

Parece evidente que la gestión colectiva de conflictos entre familias ofrece un amplio margen de negociación y práctica democrática que difícilmente se puede dar si se abordan los problemas individualmente. En la gestión colectiva de conflictos se podría llegar a situaciones donde el derecho a la privacidad y la protección de datos se viera comprometida durante el proceso de debate y consenso. En tal caso se podrían dar situaciones que conculcarían dos derechos fundamentales enfrentados (la participación en la escuela y en la educación de los hijos frente al derecho a la privacidad). Se trata de una evidente situación de complejidad pedagógica y jurídica, donde se produce un aprendizaje organizacional de ciudadanía activa. El análisis cualitativo permite detectar cierto desacuerdo entre los distintos sectores educativos, que se concreta en una posición corporativa del entorno docente (profesorado y direcciones) por abordar los conflictos individualmente, mientras que las familias están bastante más divididas en sus opiniones. Sorprende el hecho de que el sector parental sea el único que aboga por la implicación de los delegados de padres y madres del grupo, que habrían de ser los responsables de vehicular sus voces y sus postulados en el sistema escolar establecido por la normativa. Según las entrevistas mantenidas, esto parece imputable a cierta prevención que en especial las direcciones de los centros tienen contra la gestión del derecho de las familias a interactuar colectivamente con el centro docente. 
Figura 4. Respuestas de la muestra a la pregunta "Cuando existe un problema con un grupo de familias, ¿cuál es la mejor manera de abordarlo?»

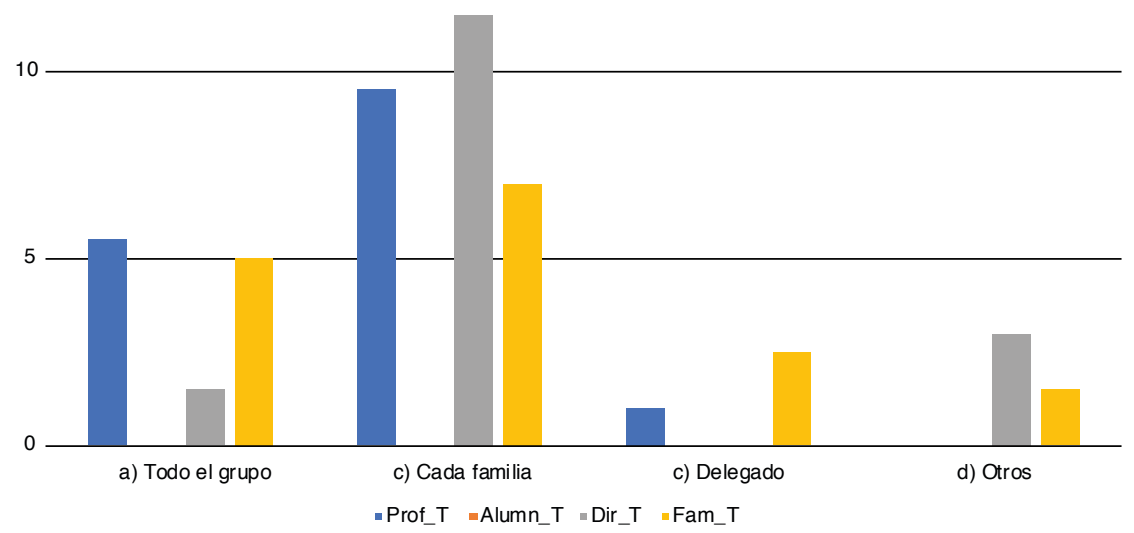

Fuente: elaboración propia.

Los resultados recogidos en esta investigación han permitido constatar la variedad de las percepciones sobre comunicación e interacciones en el seno de la organización escolar. Sobre los componentes explícitos se ha detectado el desconocimiento absoluto de muchas familias sobre los ámbitos y los temas educativos en los que pueden intervenir, tanto en las reuniones de inicio de curso como en las distintas situaciones donde hay que decidir cuestiones significativas a lo largo de la escolarización de sus hijos e hijas. Sobre la aplicación práctica y la experiencia implícita se ha de denunciar el escaso cumplimiento de las propuestas de representación democrática parental por votación, recogido en la normativa de referencia. Sobre la reflexión acerca de la propia práctica, a partir de la toma de conciencia de la gestión del conflicto y sus alternativas, se hace evidente una falta de madurez negociadora y democrática en el entorno escolar.

Por otro lado, el contraste de los cuestionarios con la información recogida en los documentos de gestión de los centros educativos demuestra cierta disonancia y contradicción en diversos centros. La autora realizó una primera recogida de datos sobre los documentos preceptivos y los analizó a través de la misma matriz de complejidad conceptual, para cuantificar la frecuencia de contenidos de cada uno de los niveles propuestos. Estos documentos se pueden considerar declaraciones de intenciones de los mandatarios de los centros docentes en relación con la ejecución de sus responsabilidades para con la Administración como servicios públicos. Esto permite conocer la forma en que una comunidad educativa se quiere organizar, sus pretensiones y su forma de abordar la educación en un contrato tácito, que debería coincidir con la realidad percibida por los diferentes agentes de la comunidad 
educativa. La triangulación posterior en los grupos de debate de cada centro ha contribuido a identificar la débil implicación de las familias en temas que requieren su participación y ha confirmado que en las escuelas donde la participación estructurada es más intensa y está organizada adecuadamente el clima de trabajo es mejor y el alumnado tiene más oportunidades para obtener buenos resultados académicos.

\section{Conclusión y reflexiones}

Esta investigación ha dedicado buena parte del estudio teórico de los conceptos analizados a la reflexión sobre problemas de univocidad y objetivación en la recogida de información, con el objeto de profundizar en el valor de una herramienta que permite pasar de conceptos subjetivos y líquidos a datos cuantificados y parametrizados que se puedan operar para automatizar cálculos y correlaciones. La virtualización educativa durante la pandemia de COVID-19 ha influido en las comunicaciones escolares más allá de cualquier formación programada y ha facilitado la investigación en línea.

La riqueza del ecosistema digital actual se compone de la agregación y la confluencia de textos y mensajes de muy diversa índole, que se complementa con una enorme cantidad de información situacional. Las variables contextuales pueden ser extremadamente precisas (por ejemplo: la identidad del autor, la geolocalización de la emisión, los proveedores de la telecomunicación, la filiación profesional o cualquier otro parámetro vinculado al proceso de transmisión de datos). La heterogeneidad de las interacciones de los agentes en las diferentes comunidades sociales es igualmente enriquecedora, y cualquier interacción que tengan con y entre los diferentes grupos de la comunidad educativa seguramente será distinta (Baferani, 2015). Las opiniones coincidentes o contradictorias entre alumnado, profesorado, familias y dirección del centro dan lugar a una gradación numérica (consenso total $=1$ ) que se puede comparar, ordenar y considerar en diferentes comunidades, espacios y tiempos. Esta sencilla triangulación permite conocer y cuantificar la cohesión de la escuela en temas muy diversos.

Por lo que a la información se refiere, los contenidos de los mensajes y de los textos son difíciles de comparar, puesto que la carga significativa de una palabra o de una frase en lenguaje natural puede ser muy distinta para cada persona ("hace frio» o "hace calor», por ejemplo, no correlaciona con una medición exacta de ninguna escala térmica objetiva). Los seres humanos constatan realidades distintas a partir de su percepción individual e intransferible (Peñaloza Páez, 2012), en lo que podríamos llamar el ADN de percepción conceptual de cada uno, distinto dentro de un grupo (Marksteiner, Nishen y Dickhäuser, 2021) y exclusivo en los grupos dentro de comunidades diversas. Son diferentes las miradas personales, comunitarias o sectoriales, y todas ellas construyen el valor colectivo cuando se agregan las percepciones de cada sujeto y de los distintos grupos de sujetos (Allport, 1954) para determinar valores, ordenaciones e índices mesurables de la realidad. 
Por todo lo expuesto, este estudio ha permitido generar, aplicar y validar una matriz conceptual que, a modo de filtro de gestión cuantitativa, identifica distintos ámbitos de complejidad cualitativa en la implicación de las familias en la comunidad educativa. El objetivo procedimental se cumple con evidencias de éxito en los distintos ámbitos estudiados, en la revisión de documentación, en la aplicación de los cuestionarios y en la negociación de futuras acciones de transformación pedagógica propuestas durante las entrevistas en los grupos de discusión.

El análisis conclusivo permite identificar hechos significativos a mejorar, que los centros escolares participantes han decidido abordar y gestionar desde una mirada innovadora y de cambio organizacional, durante los próximos cursos. En una apreciación global, se extrajeron las conclusiones siguientes:

Primero: familias y centros coinciden más en las apreciaciones sobre valores y principios que en el reglamento que han decidido compartir, en los procesos relacionales o en los mecanismos de solución de conflictos. Esto se podría atribuir a las exigencias burocráticas que fuerzan a la redacción de documentos administrativos (a menudo sin tiempo suficiente) o bien a que la redacción de los documentos, en sí misma, siendo esta a priori, adolece del registro de cambios durante su ejecución, y por tanto la realidad no se refleja en el propio documento.

Segundo: todos los equipos directivos consideran esencial la relación entre la escuela y la familia / la familia y la escuela, como se ha podido comprobar en todas y cada una de las entrevistas mantenidas con los agentes implicados, así como en el resultado de las encuestas. No obstante, muchos equipos directivos y docentes prefieren optar por tratar los asuntos educativos con cada familia por separado, en lugar de utilizar los sistemas de participación representativa de padres y madres.

Tercero: las escuelas están bien valoradas por el 90\% de las familias, aunque hay que entender que esta podría ser una apreciación sesgada. Si bien es verdad que los resultados de encuestas, entrevistas y demás apreciaciones hechas por la autora apuntan en esta dirección, también es cierto que las personas que han decidido participar en estas muestras tienden a la concordia y se sienten cómodas en el propio centro.

Cuarto: la relación del docente con las familias no está reglamentada en más del $40 \%$ de los centros educativos, cosa que debería alarmarnos y focalizar más nuestros esfuerzos de consenso, puesto que todas y cada una de las actuaciones realizadas y de las decisiones tomadas en relación con el alumno deben contar directa o indirectamente con el consentimiento parental.

A partir de estos resultados, puede afirmarse que las escuelas de la muestra deberían revisar los procesos participativos que permiten y fomentan la implicación de las familias, además de crear instrumentos para mejorar el sistema educativo desde la cohesión y la inclusión.

Si, como decía el protagonista de El Principito, de Saint-Exupéry (1943), «le langage est source de malentendus» («la lengua es fuente de malentendidos»), para entendernos bien, debemos esforzarnos en mejorar esa fuente de 
entendimiento y complicidad. La implicación de las familias en la escuela y la participación en los procesos educativos requieren una buena comunicación entre las dos instituciones. A través de ese esfuerzo compartido lograremos una escuela aún mejor.

\section{Referencias bibliográficas}

Aguilar, G.; Demosthenes, Y. y Campos, I. (2020). La participación familiar en la inclusión socioeducativa de los educandos con necesidades educativas especiales. Revista de Educación, Mendive, 18(1) (enero-marzo). Recuperado de <http://scielo. sld.cu/scielo.php?script=sci_arttext\&pid=S1815-76962020000100120>.

AlLport, G.W. (1954). The nature of prejudice. Boston, MA: Addison-Wesley Publishing Company. Recuperado de <https://faculty.washington.edu/caporaso/courses/ 203/readings/allport_Nature_of_prejudice.pdf>.

Astudillo, M.P. y ChÉvez, P. (2014). Agentes e instituciones de la educación: Una reflexión desde las desigualdades sociales. CIENCIA ergo-sum, 22(2), 161-166.

Baferani, M.H. (2015). The Role of the Family in the Socialization of Children. Mediterranean Journal of Social Sciences, 6(6 S6), 417-423. Recuperado de <https://www.richtmann.org/journal/index.php/mjss/article/view/8516>.

B Loom, B. (1956). Taxonomy of educational objectives, handbook. The cognitive domain. Nueva York: David McKay.

Dietze, P. y Knowles, E.D. (2021). Social class predicts emotion perception and perspective-taking performance in adults. Personality and Social Psychology Bulletin, $47(1), 42-56$. <https://doi.org/10.1177/0146167220914116>

EIPSI (2021). Evidence Informed Practice for School Inclusion-EIPSI. Erasmus+ Project, no. 2020-1- ES01-KA201-082328. Recuperado de <https://edtech2021.wixsite. com/eipsi>.

FernÁNDEZ BATANERo, J.M. (2012). Dirección y buenas prácticas educativas en centros de orientación educativa. En AFOE. I Congreso Internacional Virtual sobre Innovación Pedagógica y Praxis Edicativa. Innovagogía 2012. Libro de Actas (pp. 97-106). Sevilla: AFOE Formación. Asociación para la Formación, el Ocio y el Empleo. Recuperado de <https://documat.unirioja.es/descarga/libro/535470.pdfs.

Fernández-Rouco, N.; Fallas-Vargas, M.A. y García-Martínez, J.A. (2020). Voces protagónicas para el bienestar en la escuela: Análisis cualitativo de la perspectiva de los agentes implicados. Voces y Silencios: Revista Latinoamericana de Educación, 11(2), 49-66. $<$ https://doi.org/10.18175/VyS11.2.2020.3>

Garreta, J. (2012). Famílies i escoles: La participació de les famílies en els centres educatius. Lleida: Pagès Editors.

Grek, S. e YDesen, Ch. (2021). Where science met policy: Governing by indicators and the OECD's INES Pogramme. Globalisation, Societies and Education, 19(2), 122-137. <https://doi.org/10.1080/14767724.2021.1892477>

Jiménez, R. (2020). Aplicación del Modelo de Lawshe. Validez de contenido [vídeo]. Recuperado de <https://www.youtube.com/watch?v=aP917BFlhGY>.

Jeynes, W.H. (2011). Parental Involvement and Academic Success. Nueva York: Routledge. 
Kernan, M. (2012). Parental Involvement in Early Learning: A review of research, policy and good practice. La Haya: International Child Development Initiatives; Bernard van Leer Foundation. Recuperado de <https://efc.issuelab.org/resour ces/15860/15860.pdf>.

Marksteiner, T.; Nishen, A.K. y Dickhäuser, O. (2021). Students' perception of teachers' reference norm orientation and cheating in the classroom. Frontiers in Psychology, 12, 125. Recuperado de <https://www.frontiersin.org/articles/10.3389/ fpsyg.2021.614199/full>.

Martín, M. y Gairín, J. (2007). La participación de las familias en la educación: Un tema por resolver. Bordón: Revista de Pedagogía, 59(1). 113-151. Recuperado de $<$ https://recyt.fecyt.es/index.php/BORDON/article/view/36840/20439>.

Martín, M. y Muñoz, Y. (2006). La participación de los padres en educación. En M. Martín y J. Gairín SAllán. La participación en educación: Los consejos escolares, una vía de participación (pp. 183-202). Santiago de Chile: Fundación Creando Futuro.

Mateos, M. y Peñalba, G. (2003). Aprendizaje a partir de un texto científico en la universidad. En C. Monereo y J.I. Pozo (eds.). La Universidad ante nueva cultura educativa. Enseñar y aprender para la autonomía (pp. 79-92). Madrid: Ed. Síntesis / ICE UAB.

Naciones Unidas (2015). Transformar nuestro mundo: La Agenda 2030 para el Desarrollo Sostenible. Resolución aprobada por la Asamblea General el 25 de septiembre de 2015 (A/res/70/1). Recuperado de <https://www.unfpa.org/sites/default/ files/resource-pdf/Resolution_A_RES_70_1_SP.pdf $>$.

Niño, L. y GAmA, A. (2014). Las políticas educativas de competencias en la globalización: Demandas y desafíos para el currículo y la evaluación. Itinerario Educativo, 64, 37-64.

OECD (2018). Preparing Our Youth for an Inclusive and Sustainable World - The OECD PISA global competence framework. OECD. Recuperado de <http://www. oecd.org/pisa/aboutpisa/Global-competency-for-an-inclusive-world.pdf $>$.

Peñaloza PÁez, J. (2012). Los grupos sociales y nuestro papel en ellos. Contribuciones a las Ciencias Sociales, 15.

Razeto Pavez, A. (2016). Estrategias para promover la participación de los padres en la educación de sus hijos: El potencial de la visita domiciliaria. Estudios Pedagógi$\cos$ (Valdivia), 42(2), 449-462. <https://dx.doi.org/10.4067/S0718-07052016000200026>

Rivas, S. y UGarte, C. (2014). Formación docente y cultura participativa del centro educativo: Claves para favorecer la participación familia-escuela. ESE: Estudios sobre Educación, 27, 153-168. <https://doi.org/10.15581/004.27.153-168>

SAInT-Exupéry, A. DE (1943). Le petit prince. Reynal \& Hitchcock (Nueva York). Gallimard (Francia, 1945).

Somprach, K.; TAng, K.N. y PopoonsaK, P. (2017). The relationship between school leadership and professional learning communities in Thai basic education schools. Educational Research for Policy and Practice, 16(2), 157-175. <https://doi.org/10.1007/s10671-016-9206-7>

UNESCO (Organización de las Naciones Unidas para la Educación, la CienCIA Y la Cultura) (2014). Educación global para la ciudadanía mundial: Preparar a los educandos para los retos del siglo XXI. París: UNESCO Publishing. Recuperado de <https://unesdoc.unesco.org/ark:/48223/pf0000227729>. 
- (2015a). Declaración de Incheon: Educación 2030: Hacia una Educación Inclusiva y Equitativa de Calidad y un Aprendizaje a lo Largo de la Vida para Todos. París: UNESCO Publishing. Recuperado de <https:/unesdoc.unesco.org/ark:/48223/ pf0000233137_spa?posInSet=1\&queryId=c5743d57-2d51-4e8b-8819-a778f46 bd5b2>.

- (2015b). Replantear la educación: ¿Hacia un bien común mundial? París: UNESCO Publishing. Recuperado de <https://unesdoc.unesco.org/ark:/48223/pf0000 232697>.

- (2017). La Educación transforma vidas. París: UNESCO Publishing. Recuperado de <https://unesdoc.unesco.org/ark:/48223/pf0000247234_spa>.

Verger, A.; Novelli, M. y Altinyelken, H.K. (eds.) (2018). Global Education Policy and International Development: New agendas, issues, and policies. 2a ed. Londres: Bloomsbury Academic. Recuperado de <https://www.academia.edu/36434638/ Global_Education_Policy_and_International_Development_2018_second_ edition>.

Werbach, K. y Hunter, D. (2012). For the win: How game thinking can revolutionize your business. Harrisburg: Wharton Digital Press.

Wiseman, A. y Stevens Taylor, C. (eds.) (2017). The Impact of the OECD on Education Worldwide. International Perspectives on Education and Society, JoSPoE, 31. Bingley: Emerald Publishing.

Zardoya Loureda, M.V. (2015). La arquitectura educacional de los sesenta en Cuba. Revista Arquitectura y Urbanismo, 23(3). 